\title{
A Sheet-Stacking Technique for Making Multiple Air- Suspended-Core Optical Fibres
}

\author{
Jindan Shi, Xian Feng, Zhenggang Lian, Nicholas White, Peter Horak, and Wei H. Loh \\ Optoelectronics Research Centre, University of Southampton, Southampton, SO17 1BJ, U.K. \\ jxs@orc.soton.ac.uk
}

\begin{abstract}
We demonstrate using a sheet-stacking method to fabricate nanomechanical optical fibres with long and thin spokes. This approach shows great advantage for manufacturing complex multi-material speciality optical fibres with novel photonic and nanomechanical functionalities.

(C)2013 Optical Society of America

OCIS codes: 060.2280 Fiber design and fabrication; 060.4005 Microstructured fibers
\end{abstract}

\section{Introduction}

The demonstration of nanomechanical functionality in dual air-suspended-core (ASC) optical fibres, i.e., nanomechanical optical fibres, has attracted significant interest because of their many potential applications for optical sensing and optical networking systems, including switching, routing, buffering, etc [1]. So far, such ASC fibres have been fabricated using an extrusion technique, which is normally used for making non-silica glass complex structured fibres with low processing temperature [2]. However, the extrusion approach has several limitations; e.g., (i) while the use of silica glass would be beneficial for this type of novel fibre because of its ultra-low loss; silica glass also requires very high temperature $\left(>1500^{\circ} \mathrm{C}\right)$ for extrusion and few die materials work well under such high temperatures and high extrusion pressures $\left(0.1-10 \mathrm{kN} / \mathrm{cm}^{2}\right)$; (ii) the material of one core needs to be different from that of another; (iii) multiple movable ASCs (e.g. 10 cores) are required for novel photonic applications. Therefore, a new fabrication technique is highly desirable.

From the geometry of the nanomechanical optical fibre, a sheet-stacking technique, i.e., periodically repeating the element of a core on a sheet in the vertical direction, is extremely suitable for fabricating multiple ASC nanomechanical optical fibres. For the simplest case, a single ASC fibre can be drawn from a preform consisting of a core rod, a thin sheet and a surrounding thick walled jacket tube [3], as seen in Fig. 1(a). Multiple ASCs based on single or various materials can be built using this platform, as shown in Fig. (b)\&(c). When the spokes have a thickness much less and a length much longer than the optical wavelength, the light will be guided in the cores and will not leak through the spokes into the jacket. Secondly, due to the mechanical properties of the submicron-size cores and submicron thin but tens-of-micron long spokes, these cores can be easily moved by small forces, e.g., air pressure [1], electrostatic actuation [4] or even optical forces. In addition, as long as the materials employed (in particular for the cores and the sheets) are thermally compatible, various core materials can be adopted for achieving multiple functions in one fibre.
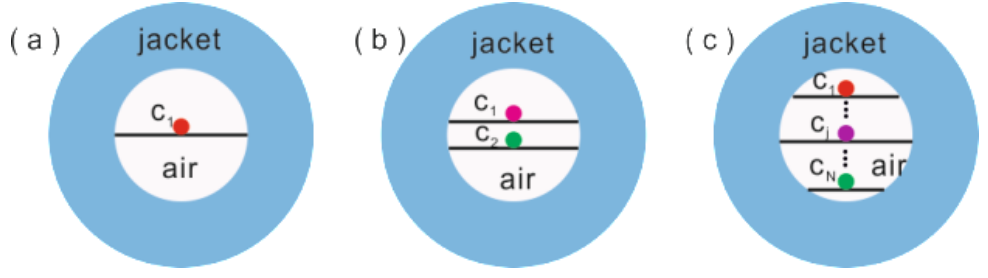

Fig.1 Schematic diagrams of using the sheet-stacking method to build (a) single-, (b) dual- and (c) N-ASC fibres

In this work, we report the fabrication of multiple ASC fibres using the sheet-stacking method. The fibre cores and spokes are based on two different borosilicate glasses. The drawn dual-ASC fibre has very thin $(\sim 140$ $\mathrm{nm})$ and long $(\sim 39 \mu \mathrm{m})$ spokes. This fibre has been successfully connected to a commercial silica fibre by fusion splicing.

\section{Fabrication and characterization of dual-ASC fibre}

We chose commercial Schott N-BK7 glass and Schott D263T glass as the materials for the core and sheet respectively. Both glasses are borosilicate glasses but with slightly different thermal properties. Fig.2(a) shows the viscosity curves of these two glasses. It is seen that the two glasses have similar viscosity behaviour. As shown in Fig.2(b), the outer walls of the preform were constructed using four pieces of N-BK7 plates, each 2 $\mathrm{mm}$ thick. Two slots were sawed at the centre of each vertical wall with $0.2 \mathrm{~mm}$ width, $1.0 \mathrm{~mm}$ depth and with $1.3 \mathrm{~mm}$ spacing between them. Two D263T sheets with a thickness of $0.1 \mathrm{~mm}$ were horizontally stacked using the slots. Two N-BK7 glass cores were then placed in the preform, one on each sheet. Each core has a rectangular cross section of $0.86 \times 0.94 \mathrm{~mm}$ and was aligned to the centre of the sheet. This stacked preform was 
then heated in a furnace at $600-700^{\circ} \mathrm{C}$ for $0.5-2$ hours to fuse all the pieces of the preform together (see Fig. 2 (b)). Graphite sheets were placed below each D263T sheet to prevent the glass sheets from sagging down during the heating. The annealed preform was then elongated to a cane of diameter $2.1 \mathrm{~mm}$. The cane was then inserted into a Schott LLF1 glass jacket tube with an outer diameter (OD) of $10 \mathrm{~mm}$ and an inner diameter (ID) of 2.2 $\mathrm{mm}$. This ensemble was finally drawn into fibre. Positive pressure was applied to the air holes during fibre drawing. As seen in Fig.2 (c), the fibre has an OD of $160 \mu \mathrm{m}$ and two cores with dimensions of $0.8 \times 2.6 \mu \mathrm{m}$. The two supporting spokes have a uniform thickness of $140 \pm 8 \mathrm{~nm}$ and a length of $39 \mu \mathrm{m}$.

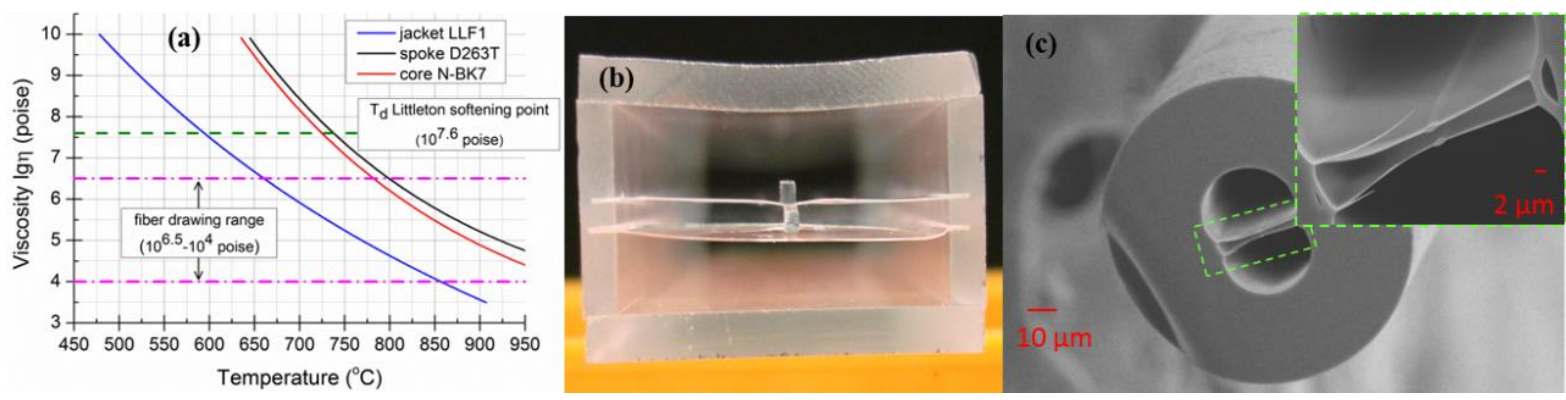

Fig.2 (a) Viscosity curves of LLF1, N-BK7 and D263T glasses. (b) Optical photograph of dual-ASC preform assembled by the sheetstacking method; (c) SEM photo of dual-ASC fibre; inset: magnified image of the central part.

Based on theoretical mode analysis, the dual-ASC fibre is multimoded at $1550 \mathrm{~nm}$, with two TE (horizontal polarization) modes and two TM (vertical polarization) modes. However, it can be operated as single-mode by adjusting the launching condition, as shown in Fig. 3(a), and holding the fibre straight. Via the cut-back method, the fibre loss was measured as $3.9 \pm 0.1 \mathrm{~dB} / \mathrm{m}$ at $1550 \mathrm{~nm}$ for both fundamental TE and TM modes. For comparison, an N-BK7 unclad fibre with an OD of $230 \pm 5 \mu \mathrm{m}$ was also drawn from a rod bulk glass and its loss was measured to be $3.9 \pm 0.05 \mathrm{~dB} / \mathrm{m}$ (Fig. 3(b)), indicating there is no additional loss resulted from the sheetstacking approach. By choosing a glass with lower material loss than N-BK7, e.g., silica as the core, multiple ASC fibres with much lower loss can be fabricated. A commercial fusion splicer was used to splice the dualASC fibre to a commercial high NA silica fibre (Nufern UHNA4, NA=0.35) by the offset fusion splicing approach [5], see Fig. 3(c). The splice loss is $\sim 2.7 \mathrm{~dB} /$ joint.

(a)

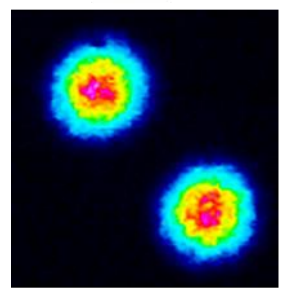

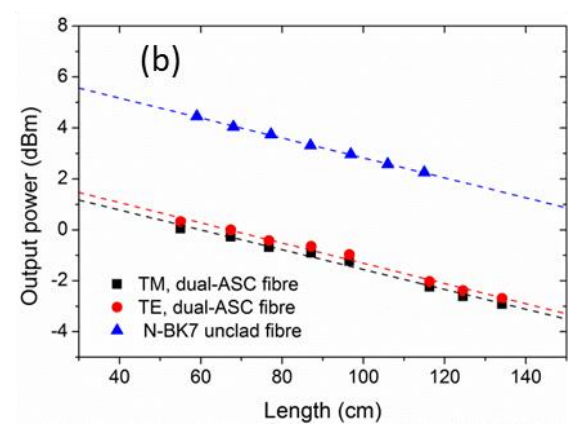

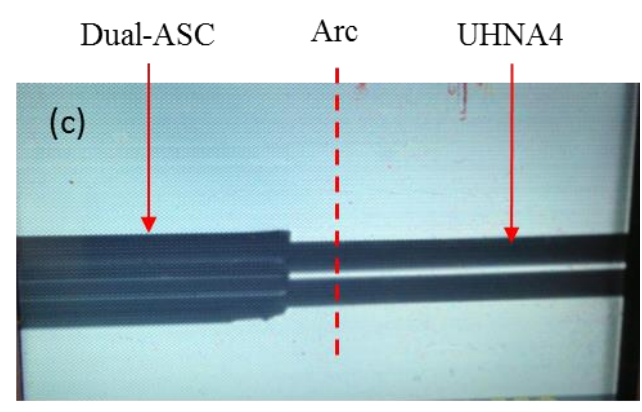

Fig.3 (a) The near-field image from the dual-ASC fibre at $1550 \mathrm{~nm}$; (b) Measured dual-ASC and N-BK7 unclad fibre losses at 1550 nm; (c) Photograph of splicing the dual-ASC fibre with UHNA4 fibre.

\section{Conclusions}

We have demonstrated the fabrication of nanomechanical optical fibres using a sheet-stacking technique. Negligible additional fibre loss has been observed from this fabrication method. Moreover, the dual-ASC fibre could be spliced to commercial silica fibre. By optimising the fabrication parameters, the core diameters, the core-to-core spacing, and the spoke thickness can be further reduced, and the spoke length can be further lengthened to tune the nanomechanical properties of the suspended cores of the multiple ASC fibre for, e.g., switching or sensing applications. This work was supported by the Engineering and Physical Sciences Research Council (EPSRC), under the EPSRC Centre for Innovative Manufacturing in Photonics.

\section{References}

1. Z. Lian, et al., "Nanomechanical optical fiber," Opt. Express 20(28), 29386-29394 (2012).

2. X. Feng, et al., “Nonsilica glasses for holey fibers," J. Lightwave Tech. 23(6), 2046-2054 (2005).

3. P. Kaiser, E. A. J. Marcatili, and S. E. Miller, “A New Optical Fiber," The Bell Syst. Tech. J. 52, 265-569 (1973).

4. N. Podoliak, et al., "Electrostatic control of dual-core optical fibre with NEMS functionality", CLEO-Europe/IQEC 2013, Munich, Germany, paper Cl-4.2 (2013).

5. X. Feng, et al., "Dispersion controlled highly nonlinear fibers for all-optical processing at telecoms wavelengths," Optical Fiber Technology, 16(6): p. 378-391 (2010). 